\title{
Signal detection without finite-energy limits to quantum resolution
}

\author{
Alfredo Luis* \\ Departamento de Óptica, Facultad de Ciencias Físicas, Universidad Complutense, 28040 Madrid, Spain
}

(Dated: January 17, 2013)

\begin{abstract}
We show that there are extremely simple signal detection schemes where the finiteness of energy resources places no limit to the resolution. On the contrary, larger resolution can be obtained with lower energy. To this end the generator of the signal-dependent transformation encoding the signal information on the probe state must be different from the energy. We show that the larger the deviation of the probe state from being minimum-uncertainty state, the better the resolution.
\end{abstract}

PACS numbers: $03.65 . \mathrm{Ca}, 03.65 . \mathrm{Ta}, 42.50 . \mathrm{St}, 42.50 . \mathrm{Dv}$

\section{INTRODUCTION}

Precise detection provides a readily practical application of quite fundamental quantum ideas, such as quantum statistics, nonclassical states, and uncertainty relations. Quantum metrology is framed by two widespread beliefs: (i) for fixed mean energy, quantum uncertainty limits the resolution in the detection of signals with unavoidable bounds valid for all system states, and (ii) these bounds depend on the energy resources employed so that better resolution requires larger amounts of energy. This is the case of the well-known standard quantum limit and Heisenberg limit [1 11].

Here we show that there are practical schemes where energy finiteness places no limit to the resolution. On the contrary, lower energy favors larger resolution. Moreover, we also show that the larger the deviation of the probe state from being minimum-uncertainty state, the better the resolution, contrary to the more intuitive idea that improved resolution would require probes with minimum uncertainty. These results may have deep implications in the development of new detection technologies free from quantum limits. This may also provide new insights in the still open vivid debate on quantum metrology limits [2 9 ].

The structure of any signal-detection scheme is quite universal. The information about some signal $\chi$ is encoded in a system state by a signal-dependent transformation $U_{\chi}=\exp (i \chi G)$ acting on a probe previously prepared in a known state $|\psi\rangle$, where $G$ is the generator of the transformation. The transformed state $U_{\chi}|\psi\rangle$ is monitored by a measurement $M$ whose outputs provide an estimator $\tilde{\chi}$ of the signal $\chi$ with some uncertainty $\Delta \tilde{\chi}$ depending in general on $\chi, G, M,|\psi\rangle$, and the data analysis followed.

In practical terms, $G$ is essentially an interaction Hamiltonian $H_{I}$ coupling the system probe to the external variables to be monitored. In an impulsive regime, the interaction Hamiltonian $H_{I}$ is so strong, and the interaction time $\tau$ so short, that the dynamics is controlled entirely by $H_{I}$. Thus the signal $\chi$ depends on the coupling between the system and the external variables and the interaction time.
Most approaches assume that the generator of the transformation coincides with the energy $H$ of the probe system in absence of signal, $G \propto H_{I} \propto H$. This is typically the case of phase shifts in harmonic oscillators generated by the number operator $G \propto a^{\dagger} a$, where $a$ is the complex amplitude operator. The importance in physics of harmonic oscillators may suggest that this is an universal link due to very fundamental quantum features such as uncertainty relations. The examples developed in this work shows that this is not so, and when $G \neq H$ the finiteness of energy resources may place no limit on the resolution.

The condition $G \neq H$ leads naturally to the so called quantum nondemolition strategies [12, 13], in which the observable $M$, in absence of signal, is a constant of the motion $[H, M]=0$. Since observable signal-induced transformations require $[G, M] \neq 0$, quantum nondemolition $[H, M]=0$ implies that $G \neq H$, which is exactly what we are looking for. There are two typical quantum nondemolition measurements: (i) linear momentum for a free particle $M=p, H \propto p^{2}$, and (ii) energy itself $M=H$, for example for an harmonic oscillator. Next we examine both possibilities from the perspective of potential ultimate quantum limits caused by finiteness of resources.

The performance improvement available when $G \neq H$ is at the heart of previous proposals of beating quantum limits by nonlinear detection schemes where, roughly speaking, $G \propto H^{k}[10]$. This has been recently confirmed experimentally [11].

\section{FREE PARTICLE}

\section{Probe state}

Let us consider that the probe system in absence of signal is the one-dimensional dynamics of a free particle. The signal is a shift of linear momentum to be inferred by a measurement of the momentum. This is to say $H=$ $p^{2}, G=x$, and $M=p$, where $x$ and $p$ are suitably scaled dimensionless position and momentum operators with $[x, p]=i$. Following some previous works 14 16 


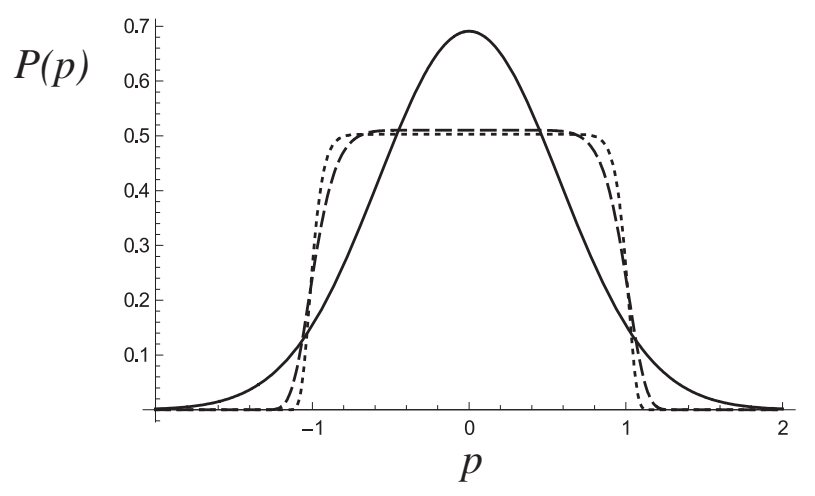

FIG. 1: Plot of the momentum statistics $P(p)$ of the initial state of the probe as a function of $p$ for $\alpha=2$ (solid line), $\alpha=10$ (dashed line), and $\alpha=20$ (dotted line), all with $\gamma$ chosen so that $\langle H\rangle=1 / 3$.

we consider the probe to be prepared in the pure state in the momentum representation

$$
\begin{gathered}
|\psi\rangle=\int_{-\infty}^{\infty} d p \psi(p)|p\rangle, \\
\psi(p)=\langle p \mid \psi\rangle=\sqrt{\frac{\alpha 2^{1 / \alpha}}{2 \gamma \Gamma(1 / \alpha)}} \exp \left(-|p / \gamma|^{\alpha}\right),
\end{gathered}
$$

where $|p\rangle$ are the momentum eigenstates, $\Gamma$ is the gamma function, and $\alpha, \gamma$ are real nonnegative parameters. The momentum statistics in absence of signal is

$$
P(p)=|\psi(p)|^{2}=\frac{\alpha 2^{1 / \alpha}}{2 \gamma \Gamma(1 / \alpha)} \exp \left(-2|p / \gamma|^{\alpha}\right) .
$$

We will consider $\alpha$ to be even integers in order to avoid continuity problems of derivatives at $p=0$. For $\alpha=2$ these are Gaussians, while for $\alpha \rightarrow \infty$ they are super Gaussians that tend to be a square distribution (see Fig. 1 ). These probes may be produced by letting free particles pass consecutively through rotating slits with suitable width and separation.

\section{Signal-induced transformation in Heisenberg and Schrödinger pictures}

The momentum shift may be created by a large force acting an extremely short time, i. e., an impulse. This force results from an interaction Hamiltonian of the form $H_{I}=-\lambda x$, where $\lambda$ is a suitable constant. In the Heisenberg picture, the equations of motion are

$$
\dot{p}=-i\left[p, H+H_{I}\right]=\lambda, \quad \dot{x}=-i\left[x, H+H_{I}\right]=2 p,
$$

with $H+H_{I}=p^{2}-\lambda x$. After an interaction time $\tau$ the transformed position $\tilde{x}$ and momentum $\tilde{p}$ in terms of the original ones $x, p$, are

$$
\tilde{p}=\lambda \tau+p, \quad \tilde{x}=\lambda \tau^{2}+2 \tau p+x
$$

where, roughly speaking, $\tau$ is given by the time spent by the particle within the interaction region. In an impulsive regime the interaction $H_{I}$ is strong compared to free energy $H$, and is acting during a time interval $\tau$ short compared with typical evolution times of the free system. Roughly speaking, $\lambda \rightarrow \infty$ and $\tau \rightarrow 0$, with finite $\chi=\lambda \tau$. In such a regime the effect of the free term $H$ is negligible and the transformation after the interaction time $\tau$ reads approximately

$$
\tilde{p}=\chi+p=U^{\dagger} p U, \quad \tilde{x} \simeq x=U^{\dagger} x U,
$$

where

$$
\chi=\lambda \tau, \quad U=\exp \left(-i H_{I} \tau\right)=\exp (i \chi x),
$$

and the signal-dependent transformation is $U=$ $\exp (i \chi G)$ with $G=x$.

On the other hand, in the Schrödinger picture the transformed probe state is

$$
\begin{aligned}
|\tilde{\psi}\rangle & =U|\psi\rangle=\int_{-\infty}^{\infty} d p \psi(p) U|p\rangle=\int_{-\infty}^{\infty} d p \psi(p)|p+\chi\rangle \\
& =\int_{-\infty}^{\infty} d p \psi(p-\chi)|p\rangle
\end{aligned}
$$

so that the momentum statistics $\tilde{P}(p)$ of the transformed probe state is

$$
\tilde{P}(p)=P(p-\chi)=\frac{\alpha 2^{1 / \alpha}}{2 \gamma \Gamma(1 / \alpha)} \exp \left(-2\left|\frac{p-\chi}{\gamma}\right|^{\alpha}\right) .
$$

\section{Examples of interaction Hamiltonian}

As a first suitable practical implementation of the desired interaction Hamiltonian $H_{I}=-\lambda x$ we may consider the interaction between a charged particle and a classical electric field $E$ in the dipole approximation with just a nonvanishing component along the $x$ axis

$$
H_{I}=-q x E, \quad \chi=q E \tau,
$$

where $q$ is the electric charge. In this case the signal $\chi$ may represent either an electric field, the charge, or the interaction time.

As a second example we may consider an Stern-Gerlach device with the interaction between a magnetic moment $\mu$ and an inhomogeneous magnetic field with just a nonvanishing component pointing in the $z$ axis $B_{z} \simeq B_{0} x$ so that

$$
H_{I}=-\mu_{z} B_{0} x, \quad \chi=\mu_{z} B_{0} \tau .
$$

In this case the signal $\chi$ may represent either the gradient of a magnetic field, a component of the magnetic moment, or the interaction time.

Within a quantum field framework, sudden momentum kicks arise from momentum conservation in the absorption/emission of photons during the interaction between the charge and the field. Thus $\chi$ represents the strength of the scattering of photons by the charge. 


\section{Signal uncertainty and main results}

Signal resolution is estimated in the standard way via the Cramér-Rao lower bound providing a minimum for the estimator uncertainty $\Delta \tilde{\chi}[17,18]$

$$
(\Delta \tilde{\chi})^{2} \geq \frac{1}{N F} \geq \frac{1}{N F_{Q}},
$$

where $N$ is the number of repetitions of the measurement, $F$ is the Fisher information

$$
F=\int_{-\infty}^{\infty} d p \frac{1}{P(p \mid \chi)}\left(\frac{d P(p \mid \chi)}{d \chi}\right)^{2}
$$

and $P(p \mid \chi)$ is the momentum statistics conditioned to the signal value $\chi . F_{Q}$ is the quantum Fisher information, that for pure states reads $F_{Q}=4(\Delta G)^{2}=4(\Delta x)^{2}$. Note that free evolution does not affect the statistics since momentum is preserved. This is a key feature of this approach as a quantum nondemolition scheme.

Since the signal-induced transformation is a momentum shift we have $P(p \mid \chi)=P(p-\chi)$ and the Fisher information does not depend on $\chi$. We readily obtain

$$
F=F_{Q}=\frac{\alpha^{2} 2^{2 / \alpha} \Gamma(2-1 / \alpha)}{\gamma^{2} \Gamma(1 / \alpha)}
$$

so that the scheme is efficient in the sense of reaching the maximum resolution allowed by the quantum Fisher information. The amount of energy involved in the measurement can be conveniently expressed by the mean value

$$
\langle H\rangle=(\Delta p)^{2}=\frac{\gamma^{2} \Gamma(3 / \alpha)}{2^{2 / \alpha} \Gamma(1 / \alpha)},
$$

where we have taken into account that $\langle p\rangle=0$.

In order to analyze the role played by finiteness of energy let us fix the value of $\alpha$, expressing $\gamma$ in terms of $\langle H\rangle$ to get a one-parameter family of probe states parametrized by $\langle H\rangle$. This allows us to get the following lower bound to the signal uncertainty

$$
(\Delta \tilde{\chi})^{2} \geq \frac{\langle H\rangle \Gamma^{2}(1 / \alpha)}{N \alpha^{2} \Gamma(2-1 / \alpha) \Gamma(3 / \alpha)} .
$$

In Fig. 2 we have represented $N(\Delta \chi)^{2} /\langle H\rangle$ as a function of $\alpha$, showing that the best scenario holds when $\alpha \gg 1$, so that the momentum statistics tends to be square. In such a case the following approximation holds

$$
(\Delta \tilde{\chi})^{2} \geq \frac{3\langle H\rangle}{N \alpha} .
$$

It can be appreciated in Fig. 2 that the approximation works quite well even for small $\alpha$.

This provides us with the main results of this work:

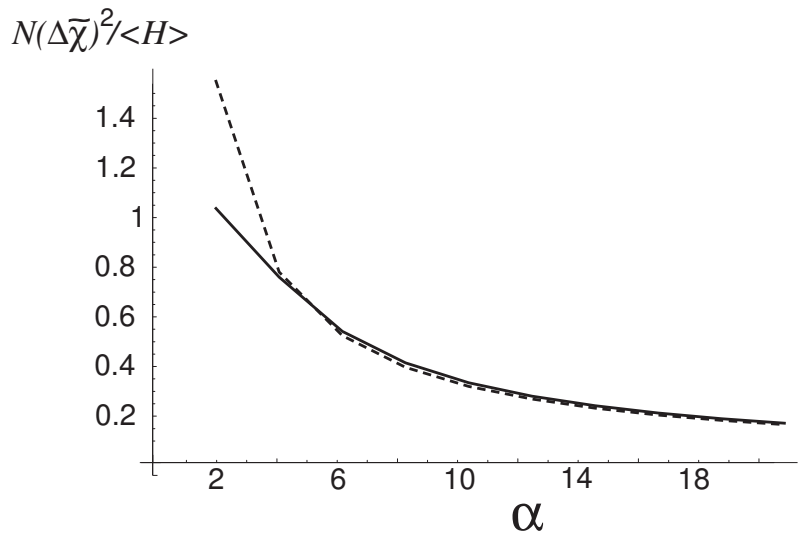

FIG. 2: Plot of $N(\Delta \tilde{\chi})^{2} /\langle H\rangle$ as a function of $\alpha$ (solid line) along with its approximation in Eq. (16) (dashed line), restricted to even values of $\alpha$.

i) The lower bound on the signal uncertainty $(\Delta \tilde{\chi})^{2}$ is proportional to $\langle H\rangle$. Thus, the lower the energy the better the resolution. This behavior can be clearly and simply explained. Roughly speaking, the inferred signal uncertainty is determined by the momentum uncertainty of the probe. For a free particle with $\langle p\rangle=0$ momentum uncertainty equals mean energy, so that lower $\langle H\rangle$ improves resolution.

ii) For fixed mean energy $\langle H\rangle$ the uncertainty (15) can be arbitrarily reduced by a proper choice of the probe state, this is simply by increasing $\alpha$ approaching a rectangular momentum statistics. This lack of limit may be ascribed to the increasingly sharp edges of $P(p)$ as $\alpha$ increases.

These results are in sharp contrast with the typical situation arising in harmonic-oscillator detection schemes, where there is an unavoidable lower bound to uncertainty inversely proportional to mean energy that applies to every probe state (see Section 3.1 below).

Finally note that for $\alpha \neq 2$ the lower bound in Eq. (15) deviates from the uncertainty $\delta \tilde{\chi}$ that may be derived from a much more simple analysis in terms of error propagation

$$
(\delta \tilde{\chi})^{2}=\frac{(\Delta M)^{2}}{N\left|\frac{\partial\langle M\rangle}{\partial \chi}\right|^{2}}=\frac{\langle H\rangle}{N} .
$$

This is because for $\alpha \neq 2$ the statistics is no longer Gaussian and the simple mean $\tilde{\chi}=\sum_{j} p_{j} / N$ need not be an efficient estimator.

\section{Numerical simulation and uncertainty relations}

The practical approaching of the lower bound depends on the number of repetitions $N$ of the measurement. An estimation of the number of repetitions $N_{B}$ required is 


\section{$P(\bar{\chi} \mid \chi)$}

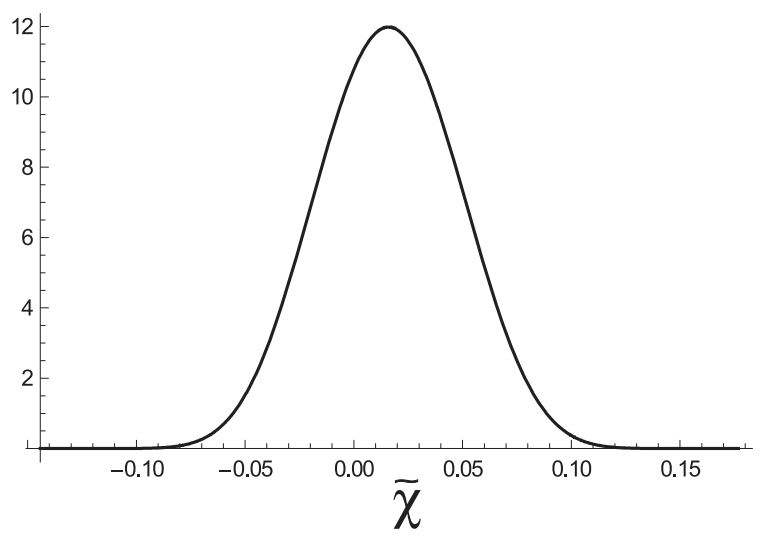

FIG. 3: Plot of a simulation of the posterior distribution $P(\tilde{\chi} \mid \chi)$ for the estimator $\tilde{\chi}$ for $\chi=0,\langle H\rangle=1 / 3, N=50$ and $\alpha=20$.

given by 17

$$
N_{B} \simeq \frac{2}{F^{2}} \int_{-\infty}^{\infty} d p\left[\frac{1}{P}\left(\frac{d^{2} P}{d p^{2}}\right)^{2}-\frac{1}{3 P^{3}}\left(\frac{d P}{d p}\right)^{4}\right]-2,
$$

leading in our case to

$$
N_{B} \simeq \frac{2 \Gamma(2-3 / \alpha) \Gamma(1 / \alpha)}{\Gamma^{2}(1-1 / \alpha)}-2 \simeq 2 \alpha
$$

where the last approximation holds for $\alpha \gg 1$. We have that the value of $N_{B}$ increases as resolution increases, but we get always accessible $N_{B}$ even for $\alpha$ values corresponding to almost perfect square momentum statistics.

A simple numerical simulation confirm the result (15). In Fig. 3 we have represented a simulation of the posterior distribution $P(\tilde{\chi} \mid \chi)$ for the signal estimator $\tilde{\chi}$

$$
P(\tilde{\chi} \mid \chi) \propto \Pi_{j=1}^{N} P\left(p_{j} \mid \tilde{\chi}\right) \propto \exp \left(-2 \sum_{j=1}^{N}\left|\frac{p_{j}-\tilde{\chi}}{\gamma}\right|^{\alpha}\right),
$$

for $\chi=0,\langle H\rangle=1 / 3$, and $\alpha=20$, conditioned to $N=50$ random outcomes $p_{j}$ of the measurement that have been simulated from an uniform distribution between -1 and 1 , which is very close to the actual distribution $P(p \mid \chi=$ 0 ) for $\alpha=20$ shown in Fig. 1. It can be appreciated that $P(\tilde{\chi} \mid \chi)$ closely resembles a Gaussian distribution. In this example the variance of $\tilde{\chi}$ in the distribution $P(\tilde{\chi} \mid \chi)$ is $(\Delta \tilde{\chi})^{2}=10^{-3}$, which coincides with the lower bound in Eq. (15) for these parameters. This coincidence is natural since after Eq. (19) the lower bound should be approached for $N_{B} \simeq 40$ repetitions.

Finally we examine the role played by the positionmomentum uncertainty relation. Taking into account that, for $\langle p\rangle=0$, we have $\langle H\rangle=(\Delta p)^{2}$ and $F_{Q}=$
$4(\Delta x)^{2}$, we can express the signal uncertainty in Eq. (11) as

$$
(\Delta \tilde{\chi})^{2} \geq \frac{\langle H\rangle}{N} \frac{1}{4(\Delta x)^{2}(\Delta p)^{2}}
$$

where $4(\Delta x)^{2}(\Delta p)^{2} \geq 1$ by uncertainty relations. Note that, contrary to what might be assumed at first sight, the minimum signal uncertainty $\Delta \tilde{\chi}$ is obtained for probe estates departing from being minimum uncertainty states. This rather paradoxical behavior was already noticed in a different context in Ref. [19].

\section{HARMONIC OSCILLATOR}

\section{Finite-energy bound for $G=x, M=p$}

For harmonic oscillators (in suitable units) $H=p^{2}+$ $\omega^{2} q^{2}$, so that neither $p$ nor $q$ are constants of the motion. Therefore, for the same conditions as above, i. e., $G=x$, $M=p$, we get very different conclusions. The mean energy, taking into account $\Delta x \Delta p \geq 1 / 2$, is

$$
\begin{aligned}
\langle H\rangle & =(\Delta p)^{2}+\langle p\rangle^{2}+\omega^{2}(\Delta x)^{2}+\omega^{2}\langle x\rangle^{2} \\
& \geq \omega^{2}(\Delta x)^{2}+\frac{1}{4(\Delta x)^{2}} \simeq \omega^{2}(\Delta x)^{2},
\end{aligned}
$$

where, since $F_{Q}=4(\Delta x)^{2}$ we consider $\Delta x \gg 1$, so that

$$
(\Delta \tilde{\chi})^{2} \geq \frac{\omega^{2}}{4 N\langle H\rangle}
$$

The dependence of $\Delta \tilde{\chi}$ on the mean energy $\langle H\rangle$ is now in the denominator, leading to an energy-depending bound that decreases for increasing energies. Moreover, the same bound holds unavoidably for every probe state with the same $\langle H\rangle$. The situation can be reverted in the freeparticle limit $\omega \rightarrow 0$, so that the approximation in Eq. (22) no longer holds and must be instead replaced by $\langle H\rangle \geq 1 /\left[4(\Delta x)^{2}\right]$.

\section{Mean number shifts}

Nevertheless, within the same harmonic-oscillator context we can provide a more sophisticated example by considering a different choice for $G$ and $M$. Let us consider that the signal is encoded via number shifts. This should correspond to a transformation generated by the phase $G=\phi$ and monitored by a number measurement $M=n$. However, the proper quantum translation of the harmonic-oscillator phase $\phi$ finds many difficulties 20], so there is no simple transformation of the form $U_{\chi}=\exp (i \chi \phi)$. To avoid this difficulty we can focus on the following transformation $U_{\chi}$ for small enough signals,

$$
U_{\chi}|n\rangle \simeq|n\rangle+\sqrt{\frac{\chi}{n+1}}|n+1\rangle
$$


where $|n\rangle$ are the eigenstates of the number operator $a^{\dagger} a$, $a^{\dagger} a|n\rangle=n|n\rangle$. This transformation produces a shift of the mean number of photons

$$
\langle n\rangle \rightarrow\langle n\rangle+\chi
$$

If the input probe state is $|n\rangle$ and the measured observable is the number operator $a^{\dagger} a$ we get that the Fisher information and the signal uncertainty are:

$$
F \simeq \frac{1}{\chi(n+1)}, \quad(\Delta \tilde{\chi})^{2} \geq \frac{\chi(\langle n\rangle+1)}{N} .
$$

We can appreciate that also within an harmonicoscillator system we can get $(\Delta \tilde{\chi})^{2} \propto\langle H\rangle$. The prize to be paid is that the example is more abstract and less practical than the free-particle case discussed in Section 2. This has been introduced here merely to illustrate that results valid for phase shifts of harmonic oscillators do not exhausts all possibilities in quantum metrology.

\section{CONCLUSIONS}

We have shown that there are simple detection schemes where resolution is not limited by finiteness of energy resources, contrary to the standard examples in harmonicoscillator systems. Moreover, we have shown that probe states deviating from minimum uncertainty provide better resolution. In practical terms this means that using schemes other than harmonic oscillators may be the key to improve resolution beyond currently established limits.

\section{Acknowledgments}

A. L. acknowledges Drs. A. Rivas and M. J. W. Hall for enlightening comments. This work has been supported by Project No. FIS2008-01267 of the Spanish Dirección General de Investigación del Ministerio de Ciencia e Innovación, and from Project QUITEMAD S2009-ESP1594 of the Consejería de Educación de la Comunidad de Madrid.

* Electronic address: alluis@fis.ucm.es

[1] V. Giovannetti, S. Lloyd, L. Maccone, Science 306 (2004) 1330-1336.

[2] A. Rivas, A. Luis, New J. Phys. 14 (2012) 093052.

[3] V. Giovannetti, S. Lloyd, L. Maccone, Phys. Rev. Lett. 108 (2012) 260405.

[4] M. J. W. Hall, D. W. Berry, M. Zwierz, H. M. Wiseman, Phys. Rev. A 85 (2012) 041802(R).

[5] M. Tsang, Phys. Rev. Lett. 108 (2012) 230401.

[6] V. Giovannetti, L. Maccone, Phys. Rev. Lett. 108 (2012) 210404.

[7] M. J. W. Hall, H. M. Wiseman, New J. Phys. 14 (2012) 033040.

[8] Y. Gao, H. Lee, J. Phys. A 45 (2012) 415306.

[9] R. Nair, arXiv:1204.3761 [quant-ph].

[10] A. Luis, SPIE Reviews 1 (2010) 018006.

[11] M. Napolitano, M. Koschorreck, B. Dubost, N. Behbood, R. J. Sewell, M. W. Mitchell, Nature 471 (2011) 486-489.

[12] V. B. Braginsky, F.Ya. Khalili, Phys. Lett. A 147 (1990) 251-256.

[13] V. B. Braginsky, F. Ya. Khalili, Rev. Mod. Phys. 68 (1996) 1-11.

[14] A. Luis, Opt. Lett. 31 (2006) 3644-3646.

[15] A. Luis, Phys. Rev. A 75 (2007) 052115.

[16] S. Zozor, M. Portesi, C. Vignat, Physica A 387 (2008) 4800-4808.

[17] S. L. Braunstein, Phys. Rev. Lett. 69 (1992) 3598-3601.

[18] S. L. Braunstein, C. M. Caves, Phys. Rev. Lett. 72 (1994) 3439-3443.

[19] D. Maldonado-Mundo, A. Luis, Phys. Rev. A 80 (2009) 063811.

[20] R. Lynch, Phys. Rep. 256 (1995) 367-436. 